\title{
Lokatis, Siegfried: Verantwortliche Redaktion. Zensurwerkstätten der DDR
}

\author{
Stuttgart: Hauswedell Verlag 2019. 576 Seiten. Preis: $€ 78$
}

\section{Reinhold Viehoff}

Online publiziert: 11. März 2020

(C) Der/die Autor(en) 2020

Es gibt wahrscheinlich neben Siegfried Lokatis keinen deutschsprachigen Buchwissenschaftler, der - als ausgewiesener Historiker - in den letzten dreißig Jahren so intensiv und umfassend Buch-, Buchhandels und Verlagsgeschichte der DDR erforscht und dazu beständig publiziert hat. Insofern war es dreißig Jahre nach dem politischen und wirtschaftlichen Zusammenbruch der DDR und ihrem Beitritt zur Bundesrepublik, gemäß einem Dezennien verbundenen Ritual, naheliegend, diese oft an weit verstreuten und nicht immer leicht zugänglichen Orten publizierten Forschungen zusammenzuführen und in einem beeindruckend umfangreichen Buch von 576 Seiten erneut vorzulegen.

Lokatis hat dieser Sammlung von Aufsätzen, die zwischen den Jahren 1993 (!) und 2017 zuerst erschienen sind, zwei bisher nicht veröffentlichte Beiträge voranund hintangestellt, die die ganze Sammlung wie eine Art Einleitung und ein Nachwort umrahmen. Die Einleitung von knappen drei Seiten also ist überschrieben mit „Der Autor ist feindlich“. Damit meint Lokatis sich selbst. Er rekurriert damit auf eine Erinnerung, die mehr aussagt als lediglich das anekdotische Format ihrer Wiedergabe. Er hatte nämlich ein frühes Manuskript zu ,Jedes Buch ein Abenteuer. Zensursystem und literarische Öffentlichkeiten in der DDR bis Ende der sechziger Jahre“ (Berlin 1997), das er gemeinsam mit Martina Langermann und Simone Barck verfasst hatte, dem letzten Leiter der Hauptverwaltung Verlage und Buchhandel in der DDR, Karlheinz Selle, nach dem Fall der Mauer zur kritischen Vorablektüre gegeben, - und bekam zurück eine Kritik, die mit dem Satz begann „Der Autor ist feindlich! Er malt alles Grau in Grau ohne positive Helden und verkennt das Typische“ (S. 7). Wer also jetzt die gesammelten Aufsätze in die Hand nimmt und 
sie noch einmal, oder zum ersten Mal, liest, kann immer dabei bedenken, dass er oder sie - aus einem bestimmten Blickwinkel gesehen - die Texte eines feindlichen Autors liest. Wenn man diese Aufsätze gelesen hat, weiß man allerdings auch, diesen bestimmten Blickwinkel genauer einzuordnen und zu verstehen. Vereinfacht - und aufs „Typische“ abgehoben - war die DDR ihrer Selbstwahrnehmung nach auf allen ihren institutionellen Handlungsebenen von Feinden umgeben, auch im Bereich des Buch- und Verlagswesens. Und im Kampf gegen diese Feinde war eben Zensur das Mittel der Wahl. Insofern ist der Untertitel des Sammelbandes „Zensurwerkstätten der DDR“ sinnvoll und Lokatis will damit ausdrücklich auch zurecht betonen, dass „Zensur in der DDR [...] kollektiv verrichtete Arbeit [war]. Sie fand in Werkstätten statt, nicht nur in der Zensurbehörde selbst, sondern auch in ihren Verlagen, die, in ein Netz streng gestaffelter Verantwortlichkeiten gespannt, integraler Bestandteil des Zensursystems waren“ (S. 8).

Die vom Autor gewählte Anordnung der gesammelten Beiträge erschließt sich nicht unmittelbar, als Lektürepfad scheint mir eher nahezuliegen, sie in der Chronologie ihrer Entstehung zu lesen. Wenn man nämlich mit dem zuerst 1993 publizierten Aufsatz „Verlagspolitik zwischen Plan und Zensur“ beginnt, sieht man, mit welcher Akribie des Aktenstudiums und historischem Rekonstruktionsvermögen Lokatis die politische Verlagspolitik von unten her aufrollt, indem er ihre wichtigsten „Widerstände, Kräfte und Schwierigkeiten“ im System selbst dingfest macht. Zu Recht weist er nämlich vor allem auf den Widerspruch hin, der dem System inhärent war: „dass das Plansystem Sachzwänge produzierte, die tendenziell politische Vorgaben konterkarierten“ (S. 37). Überhaupt weist Lokatis mit diesen - in weiteren Beiträgen immer wieder aufgezeigten - inneren Systemwidersprüchen schon früh der DDRForschung einen nicht-feindlichen Weg, das politisch durchherrschte System der DDR mit seinen organisatorischen, bürokratischen und sachlogischen Zwängen und seinem schließlichen Scheitern - zu erklären.

In dem zuerst 2017 publizierten Beitrag „Giftschränke im Leseland: Die Sperrmagazine der DDR unter besonderer Berücksichtigung der Deutschen Bücherei“ stellt Lokatis unter dem Eindruck, dass es nur dank des persönlichen Einsatzes einiger weniger - Peter Sodann in Staucha ist einer davon - gelungen sei, Millionen Bücher des Leselandes DDR vor der Vernichtung in Müllcontainern zu retten, die Frage, welche Büchern denn schon vorher dadurch symbolisch vernichtet worden seien, indem sie in Giftschränken der Bibliotheken verschwanden. Da wird dann auch das Interesse der Leser an Skurrilitäten befriedigt, an denen es in der DDR niemals ein „Defizit“ gab. Stalins Werke mussten, nachdem erst Chruschtschow den Personenkult um Stalin angeprangert und dann auch Walter Ulbricht Stalin den Wert als Klassiker abgesprochen hatte, in solchen Giftschränken verschwinden. „Doch“, so schreibt Lokatis bei der Analyse des Giftschrankes der Bibliothekarin in Eisenhüttenstadt, ,unter Breshnew kam es zu einem gewissen Revival“, nicht dem einzigen, jedenfalls ließe sich an den Büchern von und über Stalin rekonstruieren, „ob in der ideologischen Großwetterlage eine Tauwetterphase oder eine Frostperiode herrschte“" (S. 145).

Der vorliegende Sammelband ist gerade auch durch das weite Spektrum der Themen, die Lokatis behandelt, nicht nur für den interessierten DDR-Forscher immer wieder oder erneut informativ und lesenswert, sondern vielleicht ein nützliches Le- 
sebuch für Studierende - sofern in der Generation der jetzt Studierenden dreißig Jahre nach dem Ende der DDR daran noch Interesse besteht.

Funding Open Access funding provided by Projekt DEAL.

Open Access Dieser Artikel wird unter der Creative Commons Namensnennung 4.0 International Lizenz veröffentlicht, welche die Nutzung, Vervielfältigung, Bearbeitung, Verbreitung und Wiedergabe in jeglichem Medium und Format erlaubt, sofern Sie den/die ursprünglichen Autor(en) und die Quelle ordnungsgemäß nennen, einen Link zur Creative Commons Lizenz beifügen und angeben, ob Änderungen vorgenommen wurden.

Die in diesem Artikel enthaltenen Bilder und sonstiges Drittmaterial unterliegen ebenfalls der genannten Creative Commons Lizenz, sofern sich aus der Abbildungslegende nichts anderes ergibt. Sofern das betreffende Material nicht unter der genannten Creative Commons Lizenz steht und die betreffende Handlung nicht nach gesetzlichen Vorschriften erlaubt ist, ist für die oben aufgeführten Weiterverwendungen des Materials die Einwilligung des jeweiligen Rechteinhabers einzuholen.

Weitere Details zur Lizenz entnehmen Sie bitte der Lizenzinformation auf http://creativecommons.org/ licenses/by/4.0/deed.de.

Dr. Reinhold Viehoff ist Professor für Medien- und Kommunikationswissenschaft (emeritus) am Institut für Musik, Medien- und Sprechwissenschaften der Martin-Luther-Universität Halle-Wittenberg 